\title{
Evaluation of S100B in the diagnosis of suspected intracranial hemorrhage after minor head injury in patients who are receiving platelet aggregation inhibitors and in patients 65 years of age and older
}

\author{
*Heinrich Wolfgang Thaler, MD, ${ }^{1}$ Jochen Schmidsfeld, MD, ${ }^{2}$ Michael Pusch, MD, ${ }^{1}$ \\ Simon Pienaar, MD, Jörg Wunderer, MD, ${ }^{1}$ Paul Pittermann, MD, ${ }^{1}$ Rosmarie Valenta, MD, ${ }^{3}$ \\ Andreas Gleiss, PhD, ${ }^{4}$ Christian Fialka, MD, ${ }^{1}$ and Mehdi Mousavi, MD ${ }^{2}$
} ${ }^{1}$ Trauma Hospital Meidling; ${ }^{2}$ Department of Traumatology, Donauspital; and ${ }^{3}$ Department of Radiology, Kaiser-Franz-Josef-Spital,
Vienna; and ${ }^{4}$ Center for Medical Statistics, Informatics, and Intelligent Systems, Medical University of Vienna, Austria

\begin{abstract}
OBJECT Cranial CT (CCT) scans and hospital admission are increasingly performed to rule out intracranial hemorrhage in patients after minor head injury (MHI), particularly in older patients and in those receiving antiplatelet therapy. This leads to high radiation exposure and a growing financial burden. The aim of this study was to determine whether the astroglial-derived protein S100B that is released into blood can be used as a reliable negative predictive tool for intracranial bleeding in patients after $\mathrm{MHI}$, when they are older than 65 years or being treated with antiplatelet drugs (low-dose aspirin, clopidogrel).

METHODS The authors conducted a prospective observational study in 2 trauma hospitals. A total of 782 patients with MHI (Glasgow Coma Scale Score 13-15) who were on medication with platelet aggregation inhibitors (PAls) or were age 65 years and older, independent of antiplatelet therapy, were included. Clinical examination, bloodwork, observation, and CCT were performed in the traumatology emergency departments. When necessary, patients were admitted and observation took place on the ward; in these patients, CCT was performed during their hospital stay. Patients with severe trauma, focal neurological deficits, posttraumatic seizures, anticoagulant therapy, alcohol intoxication, coagulation disorder, blood sampling more than 3 hours after trauma, and unknown time of the trauma were excluded from the study. The median age of the patients was 83 years, and $69 \%$ were female. Sensitivity, specificity, and positive and negative predictive values of $\mathrm{S} 100 \mathrm{~B}$ with reference to CCT findings were calculated. The cutoff of S100B was set at $0.105 \mu \mathrm{g} / \mathrm{L}$.
\end{abstract}

RESULTS Of the 782 patients, 50 (6.4\%) had intracranial bleeding. One patient with positive results on CCT scan showed an S100B level below $0.105 \mu \mathrm{g} / \mathrm{L}$. Of all patients, 33.1\% were below the cutoff. S100B showed a sensitivity of $98.0 \%$ (Cl 89.5\%-99.7\%), a negative predictive value of $99.6 \%(\mathrm{Cl} 97.9 \%-99.9 \%)$, a specificity of $35.3 \%(\mathrm{Cl} 31.9 \%-$ $38.8 \%)$, and a positive predictive value of $9.4 \%(\mathrm{Cl} 7.2 \%-12.2 \%)$.

CONCLUSIONS Levels of S100B below $0.105 \mu \mathrm{g} / \mathrm{L}$ can accurately predict normal CCT findings after MHI in older patients and in those treated with PAls. Combining conventional decision criteria with measurement of S100B can reduce the CCT scan and hospital admission rates by approximately $30 \%$.

http://thejns.org/doi/abs/10.3171/2014.12.JNS142276

KEY WORDS minor head injury; intracranial hemorrhage; S100B; platelet aggregation inhibitors; older patients; traumatic brain injury

\footnotetext{
ABBREVIATIONS CCT = cranial CT; ED = emergency department; $\mathrm{GCS}=$ Glasgow Coma Scale; $I \mathrm{QR}=$ interquartile range; $\mathrm{MHI}=$ minor head injury; $\mathrm{NPV}=$ negative predictive value; $\mathrm{PAI}=$ platelet aggregation inhibitor; $\mathrm{PPV}=$ positive predictive value .

SUBMITTED October 3, 2014. ACCEPTED December 12, 2014.

INCLUDE WHEN CITING Published online July 7, 2015; DOI: 10.3171/2014.12.JNS142276.

DISCLOSURE This study was supported by a grant from the scientific fund of the Allgemeine Unfallversicherungsanstalt (AUVA-the Austrian Workers' Compensation Board); research account Nr FK 19/12, Medical Head Office, AUVA.

* Drs. Thaler and Schmidsfeld contributed equally to this work.
} 
I $\mathrm{N}$ adults age 65 years and older, head injuries are frequently observed. As the aging population rapidly grows, the number of these mostly fall-related injuries increases at an even higher rate. ${ }^{8,30} \mathrm{Up}$ to $90 \%$ of all treated head injuries are minor head injuries (MHIs), defined by a score of 13-15 on the Glasgow Coma Scale (GCS). ${ }^{29,36}$ They occur with an incidence of 60-300 per $100,000 .^{11}$ Intracranial bleeding is detected in approximately $7 \%$ of those patients, and they very rarely require neurosurgical intervention. ${ }^{1,9,13,16}$ To rule out intracranial hemorrhage, patients with $\mathrm{MHI}$ must undergo cranial CT (CCT) scans, and many of them are additionally hospitalized for observation. The decision criteria used in the management of $\mathrm{MHI}$ are not standardized and remain controversial. ${ }^{9,13,18,27,33}$

In older patients and in patients on platelet aggregation inhibitors (PAIs), i.e., low-dose aspirin and clopidogrel, the common clinical practice is to perform a CCT scan, often combined with clinical observation, because these groups are assumed to have a higher rate of intracranial bleeding. ${ }^{4,6,15,28}$ In addition, they are also considered to suffer from delayed bleeding. ${ }^{20,28}$ Therefore, the rate of hospitalization more than doubles for patients age 65 years and older, compared with the general population with head injuries. ${ }^{30}$ This is not only problematic with regard to the radiation exposure, but also represents an enormous financial burden. ${ }^{24}$

In the last 20 years, evidence has shown that measurement of the protein S100B in serum has been useful in the management of MHI. 2,12,14,17,22,32,34,38 S100B is a calciumbinding peptide that is mainly produced by astrocytes. Increased S100B concentrations after brain trauma are a consequence of the destruction of astrocytes combined with a temporary dysfunction of the blood-brain barrier. ${ }^{21}$ Together with the conventional clinical decision criteria, measurement of S100B levels can be used safely to exclude approximately $30 \%$ of adult patients from further diagnostic procedures like CCT if the blood sample is drawn within 3 hours after injury. 2,14,17,34 This maximum time span is based on the short half-life of S100B (90 minutes). ${ }^{21,31}$ To date, the use of S100B as a screening tool to help exclude intracranial hemorrhage in the group of patients with MHI who are older than 65 years and in patients with MHI who are receiving PAI treatment has not been specifically examined. The aim of this study was to analyze whether $\mathrm{S} 100 \mathrm{~B}$ is a reliable negative predictive tool for intracranial bleeding after MHI in older age (older than 65 years) and in patients receiving PAIs. If so, the number of CCT scans and hospital admissions for observation could be significantly reduced.

\section{Methods \\ Study Design}

This prospective, observational study was conducted from May 2011 to October 2013 at 2 trauma centers in Vienna, Austria: the Trauma Center of Meidling and the Department of Traumatology at the Donauspital. The study protocol was approved by the ethics committees of the 2 participating centers. Informed consent was obtained for all patients in the emergency department (ED). For those with GCS scores between 13 and 14, consent was obtained from the next of kin before admission. Informed consent for all patients was obtained within half an hour after arriving in the $\mathrm{ED}$, and those who met the inclusion criteria were included in the study. Inclusion criteria were as follows: MHI (GCS Score 13-15) in patients on medication with PAI who were older than 18 years, and MHI in patients age 65 years and older independent of PAI intake. Patients with MHI and severe trauma (open fractures, large open wounds, clinical signs of basal skull fracture, polytrauma), focal neurological deficits, posttraumatic seizures, anticoagulant therapy (vitamin $\mathrm{K}$ antagonists, direct oral anticoagulants), alcohol intoxication, and with a history of a coagulation disorder were excluded. Additional exclusion criteria were blood sampling more than 3 hours after index event, unknown point in time of the trauma, and missing informed consent.

\section{Patient-Related Data}

The following patient-related data were collected: age, sex, GCS score, traumatological findings, circumstances of trauma, preexisting medical conditions, medication, laboratory findings (serum glucose, serum creatinine, C-reactive protein, hemoglobin, thrombocyte count, prothrombin time, and S100B), CCT findings, interval between trauma and blood draw, interval between trauma and CCT scan, and interval between trauma and hospital discharge. The reported dosing regimen for aspirin was $100 \mathrm{mg}$ once daily, and for clopidogrel it was $75 \mathrm{mg}$ once daily. The data were collected with a standardized protocol.

All patients were seen first in the traumatology ED by surgeons who determined the GCS score and performed a brief neurological examination. Patients with MHI who were receiving antiplatelet medication or who were older than 65 years were either admitted or observed for at least 6 hours. All patients underwent CCT. The decision whether a patient had to be admitted and the point in time at which CCT was performed depended on the clinical findings. When informed consent was given, a venous blood sample was drawn within 3 hours after injury and the S100B level was determined. The result of S100B analysis was not known to the attending physician. All clinical assessments were completed before the CCT scans were performed. The CCT findings and S100B results were included in the study database by different persons and at different points in time to avoid any influence. All patients were treated as they normally would have been, following the standard operating procedures of our institutions.

\section{Cranial CT}

For CCT diagnosis both study centers used multidetector computer tomography units (Siemens Somatom Open or Siemens Definition) with 3-mm slice thickness. Patients were classified into 2 groups: CCT negative (patients with MHI who had no signs of trauma-related intracranial bleeding) and CCT positive (patients with MHI who had at least 1 trauma-related intracranial hemorrhage: i.e., epidural, subdural, subarachnoidal, or intracerebral bleeding). Each CCT scan was interpreted by a consultant radiologist 
who was blinded to the S100B level. The CCT results were spot-checked $(25 \%)$ by an independent, experienced radiologist who was blinded to patient data.

\section{Measurement of S100B}

Blood was drawn from all patients and laboratory measurements were performed immediately. The time interval between the index event and the blood draw was limited to 3 hours, according to the manufacturer's recommendation; this time period is based on the half-life of about 90 minutes for the S100B molecule. ${ }^{21,31}$ All blood specimens were processed to serum. The serum S100B levels were determined by electrochemiluminescence immunoassay (Elecsys S100; Roche Diagnostics) on the Roche cobas e601 and cobas e411 instruments. The analytical range was $0.005-39 \mathrm{mg} / \mathrm{L}$. The cutoff was set at $0.105 \mu \mathrm{g} / \mathrm{L}$ as stipulated by the manufacturer.

\section{Statistical Analysis}

The sample size for this study was chosen such that the Wilson 95\% confidence interval for the negative predictive value (NPV) would be sufficiently narrow. For the cases of one or two false-negative results, the lower confidence limit should be above $97 \%$. This could be reached by targeting at least 750 evaluable patients (based on an estimate of the rate of patients with S100B $<0.105 \mu \mathrm{g} / \mathrm{L}$ from the first 400 patients). Continuous variables are described by the median and interquartile range (IQR) due to their skewed distributions. Categorical variables are described by absolute frequencies and percentages and compared between groups by using chi-square tests. A trend of S100B across GCS categories was tested using a linear contrast with respect to log-transformed S100B. Sensitivity, specificity, positive predictive value (PPV), and NPV are reported with $95 \%$ Wilson confidence intervals. The area under the receiver operating characteristic curve is given with Wald confidence intervals. All calculations were done using SAS 9.4 software. Statistical tests were performed at a 2 -sided 5\% significance level.

\section{Results}

A total of 987 consecutive patients with MHI were considered for inclusion. In 192 patients the interval between trauma and blood sampling was more than 3 hours, and in 13 patients no CCT was performed; 782 patients therefore fulfilled all inclusion criteria. The demographic data and clinical characteristics are given in Table 1. The median age of the patients was 83 years (overall range 39-104 years, IQR $74-88$ years), and $68.7 \%$ of the patients were female.

The median interval between event and blood drawing was 2:05 hours (IQR 1:30-2:30). The median interval between trauma and CCT was 15:40 hours (IQR 3:5421:30). The median observation period was 31:37 hours (IQR 18:04-63:10). The predominant cause of trauma was a ground-level fall, followed by fall from a height. Traffic accidents were rare, and assaults were extremely rare. Most of our patients showed physical evidence of trauma above the clavicles. Nearly all patients had 1 or more preexisting medical conditions, like hypertension, chronic
TABLE 1. Demographic and clinical characteristics in 782 patients with MHI

\begin{tabular}{|c|c|}
\hline Characteristic & Value \\
\hline \multicolumn{2}{|l|}{ Median age in yrs (IQR) } \\
\hline Overall & $83(74-88)$ \\
\hline Patients w/ PAI & $83(74-88)$ \\
\hline Patients w/o PAI & $82(73-89)$ \\
\hline \multicolumn{2}{|l|}{ Age \& PAl status } \\
\hline$\geq 65$ yrs w/ PAl & $570(72.9 \%)$ \\
\hline$\geq 65$ yrs w/o PAl & $151(19.3 \%)$ \\
\hline$<65$ yrs w/ PAI & $61(7.8 \%)$ \\
\hline \multicolumn{2}{|l|}{ Sex } \\
\hline Male & $245(31.3 \%)$ \\
\hline Female & $537(68.7 \%)$ \\
\hline \multicolumn{2}{|l|}{ Trauma history } \\
\hline Fall on ground level & $653(83.5 \%)$ \\
\hline Fall from height & $58(7.4 \%)$ \\
\hline Traffic accident & $27(3.5 \%)$ \\
\hline Other & $28(3.6 \%)$ \\
\hline Unknown & $16(2.0 \%)$ \\
\hline \multicolumn{2}{|l|}{ GCS score } \\
\hline 13 & $48(6.1 \%)$ \\
\hline 14 & $147(18.8 \%)$ \\
\hline 15 & $587(75.1 \%)$ \\
\hline \multicolumn{2}{|l|}{ PAls } \\
\hline Aspirin & $526(67.3 \%)$ \\
\hline Clopidogrel & $68(8.7 \%)$ \\
\hline Aspirin + clopidogrel & $37(4.7 \%)$ \\
\hline None & $151(19.3 \%)$ \\
\hline
\end{tabular}

heart disease, diabetes mellitus, chronic obstructive pulmonary disease, osteoporosis, dementia, and Parkinson disease. Most of the patients were taking 2 or more drugs.

Of the 782 patients included, $732(93.6 \%)$ proved to be CCT negative and 50 (6.4\%) showed intracranial bleeding on CCT scans. Six hundred thirty-one patients (80.7\%) were being treated with PAIs. The patient group without PAIs, who by definition of our inclusion criteria were 65 years and older, had a median age of 82 years (IQR 73-89). The patient group with PAIs had a median age of 83 years (IQR 74-88). Of the 631 patients on PAIs, $526(83.3 \%)$ were taking low-dose aspirin, 68 (10.8\%) were on clopidogrel, and 37 (5.9\%) were taking both drugs. The respective number of intracranial hemorrhages was $32(6.1 \%)$ in the aspirin group, $3(4.4 \%)$ in the clopidogrel group, and $2(5.4 \%)$ in the group on both drugs. Patients treated with PAIs had a lower rate of intracranial bleeding (5.9\%) than those without PAIs (8.6\%). This difference, however, is not statistically significant $(\mathrm{p}=0.215)$ (Table 2$)$.

Of the 782 patients, $587(75.1 \%)$ had a GCS score of 15, $147(18.8 \%)$ a GCS score of 14 , and $48(6.1 \%)$ were graded with a GCS score of 13. The number of CCT-positive patients was $23(3.9 \%)$ in the GCS 15 group, 17 patients $(11.6 \%)$ in the GCS 14 group, and 10 patients (20.8\%) in 
TABLE 2. Rates of intracranial bleeding in relation to PAl intake in 782 patients with $\mathrm{MHI}^{*}$

\begin{tabular}{lcc}
\hline \multicolumn{1}{c}{ PAl Intake } & No. of Patients & $\begin{array}{c}\text { No. (\%) of Intracranial } \\
\text { Hemorrhages }\end{array}$ \\
\hline Aspirin & 526 & $32(6.1 \%)$ \\
\hline Clopidogrel & 68 & $3(4.4 \%)$ \\
\hline Aspirin + clopidogrel & 37 & $2(5.4 \%)$ \\
\hline All PAls & 631 & $37(5.9 \%)$ \\
\hline No PAl & 151 & $13(8.6 \%)$ \\
\hline
\end{tabular}

* There was no significant difference between patients with and without antiplatelet medication in regard to the rate of intracranial bleeding $(p=0.215)$.

the GCS 13 group (Table 3). The GCS score also correlated well with the S100B levels of these groups, showing higher values with worsening clinical symptoms ( $p$ $<0.001)$. There were 192 patients $(24.6 \%)$ who presented with a history of loss of consciousness or posttraumatic amnesia.

In the CCT-positive group, 24 patients were diagnosed with subdural, 14 with intracerebral, and 12 with subarachnoid hematoma; no epidural hematoma was found (Table 4). Four of the 50 patients with hemorrhage presented with a skull fracture. No patient required a neurosurgical intervention. The median serum S100B concentration was $0.285 \mu \mathrm{g} / \mathrm{L}$ (IQR $0.185-0.532$ ) in this group. One of 50 patients with intracranial bleeding showed an S100B concentration below the cutoff value of $0.105 \mu \mathrm{g} / \mathrm{L}$. This patient, a 95-year-old woman, had a nonfatal, small intracerebral hemorrhage near the cortex. Her GCS score was 15 and she had taken aspirin. The S100B serum concentration was $0.094 \mu \mathrm{g} / \mathrm{L}$, the blood sample was taken 75 minutes after the trauma, and CCT scanning was performed 9 hours after the injury. In control CCT scans obtained 3 days after the event, the lesion had disappeared.

In the CCT-negative group, 35.3\% showed S100B concentrations below the cutoff value of $0.105 \mu \mathrm{g} / \mathrm{L} ; 64.7 \%$ were at or above the cutoff value. The median serum S100B concentration was $0.143 \mu \mathrm{g} / \mathrm{L}$ (IQR 0.085-0.274) in this group.

The S100B results in the CCT-positive and CCTnegative groups are reported in Table 5. Calculation of sensitivity and specificity yielded the following results: 98.0\% sensitivity (CI 89.5\%-99.7\%), NPV $99.6 \%$ (CI 97.9\%-99.9\%); $35.3 \%$ specificity (CI 31.9\%-38.8\%), PPV 9.4\% (CI 7.2\%-12.2\%). Of all 782 patients, 259 (33.1\%) showed an S100B value below the cutoff of $0.105 \mu \mathrm{g} / \mathrm{L}$. The area under the receiver operating characteristic curve with respect to the radiological findings amounts to 0.73 (CI 0.67-0.79, $\mathrm{p}<0.001$ ).

\section{Discussion}

According to the recommendations from Haydel et al. (the so-called New Orleans Criteria), the "Canadian CT Head Rule," and the "Scandinavian Guidelines for Initial Management of Minimal, Mild, and Moderate Head Injuries," specific indicators exist to identify patients after MHI who should undergo CCT scanning. $7.9,13,18,27,33$ Two indicators in the patients' history in particular-age great-
TABLE 3. GCS, S100B, and CCT findings in 782 patients with MHI

\begin{tabular}{ccccc}
\hline $\begin{array}{c}\text { GCS } \\
\text { Score* }^{*}\end{array}$ & $\begin{array}{c}\text { CCT } \\
\text { Negative }\end{array}$ & $\begin{array}{c}\text { CCT } \\
\text { Positive }\end{array}$ & Total & $\begin{array}{c}\text { Median S100B Values } \\
(\text { IQR })\end{array}$ \\
\hline 13 & $38(79.2 \%)$ & $10(20.8 \%)$ & 48 & $0.284 \mu \mathrm{g} / \mathrm{L}(0.130-0.652)$ \\
\hline 14 & $130(88.4 \%)$ & $17(11.6 \%)$ & 147 & $0.178 \mu \mathrm{g} / \mathrm{L}(0.102-0.311)$ \\
\hline 15 & $564(96.1 \%)$ & $23(3.9 \%)$ & 587 & $0.139 \mu \mathrm{g} / \mathrm{L}(0.085-0.267)$ \\
\hline Total & $732(93.6 \%)$ & $50(6.4 \%)$ & 782 & $0.150 \mu \mathrm{g} / \mathrm{L}(0.088-0.291)$ \\
\hline
\end{tabular}

* GCS scores correlate well with S100B levels and with the rate of intracranial hemorrhage $(p<0.001)$.

er than 65 years and antiplatelet therapy-lead, regardless of the trauma-associated clinical aspects, to an overuse of CCT scans and observation.

The unacceptable increase of CCT and hospital admissions due to the use of these criteria was one of our reasons to perform this study. In this regard, the financial burden arising from the high CCT rates and the cost of hospital admission for observation, as well as the radiation-associated risks, are of major importance. ${ }^{3,24,25,37}$

The economic impact of S100B as a screening test in the ED management of MHI in adult patients is discussed in detail by Ruan et al. ${ }^{24}$ In that paper it was demonstrated that for S100B to be economical, more than $78 \%$ of patients with MHI must have undergone CCT. This is definitely the case in our specific patient group. Our study was performed in a central European urban region, in 2 large trauma hospitals, where the baseline rate of scanning performed in adult patients after MHI is relatively high. S100B could also be useful in rural hospitals where no CT scan is available and in the management of ED overcrowding.

Radiation from CT scans contributes to about $2 \%$ of all cancers in the US. ${ }^{3}$ Additionally, repeated exposure to CCT is associated with an increased risk of cataracts. ${ }^{37}$ Many older patients are "frequent fallers" and, after each fall leading to hospital admission, radiography and/or CT scans are frequently performed. ${ }^{23}$ Therefore, reducing unnecessary radiation exposure is a major health concern in the future.

The introduction of S100B as a reliable, examinerindependent parameter to improve the indication for CCT scans has already been recommended, among other suggestions, in the above-mentioned Scandinavian Guidelines in patients after MHI ${ }^{33}$ In patients with a GCS score of 14 and no risk factors (like anticoagulant therapy or age older than 65) or GCS 15 with loss of consciousness or vomiting and no other risk factors, S100B should be used.

Of the 782 patients, $50(6.4 \%)$ had positive CCT scans, a finding that was-surprisingly-consistent with the results of other studies in which most of the patients included were younger than 60 years. ${ }^{2,38}$ Additionally, about $80 \%$ of our patients were on medication with PAIs. In our study, older patients who had received PAIs had an even lower rate of intracranial hemorrhage than those who had not received PAIs. The difference is not statistically significant $(5.9 \%$ vs $8.6 \%$ ), but it confirms the results of the study by Spektor et al. showing no statistically significant difference in the frequency of intracranial bleeding between patients who had received low-dose aspirin and those 
TABLE 4. Intracranial injuries and CCT findings in 50 patients with bleeding from $\mathrm{MHI}$

\begin{tabular}{ll}
\hline Traumatological CCT Findings & No. $(\%)$ \\
\hline Epidural hemorrhage & $0(0.0 \%)$ \\
\hline Subdural hemorrhage & $24(48.0 \%)$ \\
\hline Subarachnoid hemorrhage & $12(24.0 \%)$ \\
\hline Intracerebral hemorrhage & $14(28.0 \%)$ \\
\hline Total & 50 \\
\hline
\end{tabular}

without. ${ }^{26}$ The similar rate of patients with positive CCT scans in our special cohort compared with other studies is a strong argument against the common assumption that older patients and those receiving PAIs have a higher risk for intracranial bleeding than younger patients and those without PAIs. Therefore, the usual clinical practice, which is to perform CCT after MHI, particularly in all older patients and in all patients treated with PAIs, should be reconsidered. In this context, the recommendation from the Scandinavian Guidelines that patients with MHI whose GCS score is $14-15$ and who present with the combination of age older than 65 years and antiplatelet medication should undergo CCT, has to be rethought or clarified.

There is an ongoing medical discussion whether a socalled "early" CCT scan is reliable in diagnosing intracranial hemorrhage after MHI, particularly in older patients with antiplatelet therapy. ${ }^{20,28}$ We regard a CCT performed within the first 3 hours after the injury as an "early" CCT. In a prospective analysis of older patients with MHI who were taking low-dose aspirin, Tauber et al. found, in patients with initially negative CCT, a $4 \%$ rate of delayed hemorrhage on repeat CCT. ${ }^{28}$ In our study an "early" CCT (within the first 3 hours after the trauma) was performed in 128 of the 782 patients (16.4\%); CCT was positive in 23 and negative in 105 . We made a sensitivity analysis in which we classified the negative CCT scans that were obtained within the first 3 hours after the event as "unverified" instead of "negative." This analysis was performed using the method proposed by $\mathrm{He}$ and McDermott and using bias-corrected and accelerated bootstrap confidence intervals. ${ }^{5,10}$ The results of the S100B test using the proposed cutoff remained quite similar after applying this statistical method in our study: NPV 99.6\% (CI 98.8\%$100 \%$ ), PPV $11.5 \%$ (CI 8.9\%-14.3\%), sensitivity $98.3 \%$ (CI $94.6 \%-100 \%$ ), and specificity $35.8 \%$ (CI $32.0 \%-39.6 \%$ ). We can conclude that the results presented in our primary analysis are not significantly influenced by the fact that our CCT scans were performed both as "early" and "late" scans. To avoid further discussion about the time point of a reliable CCT scan after trauma, we also extrapolated our results by defining CCTs performed within the first 12 hours as "unverified." This analysis yielded the same NPV of $99.6 \%$ (CI $98.3 \%-100 \%$ ).

The objective of our study was to help physicians performing the initial management of MHI in older patients decide which patients should undergo CCT, which should be admitted, and which should be discharged. For the first time, a brain biomarker, S100B, can be considered in the difficult process of evaluation and decision making
TABLE 5. S100B and CCT findings in 782 patients with MHI*

\begin{tabular}{ccccc}
\hline S100B Level & $\begin{array}{c}\text { CCT } \\
\text { Negative }\end{array}$ & $\begin{array}{c}\text { CCT } \\
\text { Positive† }\end{array}$ & Total & Predictive Value \\
\hline $\begin{array}{c}<0.105 \mu \mathrm{g} / \mathrm{L} \\
\text { (negative) }\end{array}$ & 258 & 1 & 259 & $\begin{array}{c}\text { NPV 99.6\% } \\
\text { (Cl 97.9\%-99.9\%) }\end{array}$ \\
\hline $\begin{array}{c}\geq 0.105 \mu \mathrm{g} / \mathrm{L} \\
\text { (positive) }\end{array}$ & 474 & 49 & 523 & $\begin{array}{c}\text { PPV 9.4\% } \\
(\mathrm{Cl} \mathrm{7.2 \% -12.2 \% )}\end{array}$ \\
\hline Total no. & 732 & 50 & 782 & \\
\hline
\end{tabular}

* S100B proves to be a good negative predictor for intracranial hemorrhage after MHI in patients older than 65 years and in patients being treated with PAls.

† For negative CCT findings, specificity was 35.3\% (Cl 31.9\%-38.8\%); for positive CCT findings, sensitivity was $98 \%$ (Cl $89.5 \%-99.7 \%)$.

regarding older patients and in those with antiplatelet therapy. Using a cutoff of $0.105 \mu \mathrm{g} / \mathrm{L}$ and an interval of less than 3 hours between trauma and blood drawing, S100B has been shown to be especially suited to predict the absence of pathology on CCT. The only patient observed in this study with an intracranial hemorrhage and negative S100B had a concentration slightly below the cutoff $(0.094 \mu \mathrm{g} / \mathrm{L})$ and no need of neurosurgical intervention. The NPV of S100B is $99.61 \%$. This negative prediction is of particular importance because all the other risk factors are of a positive predictive nature. In combination with conventional clinical decision criteria, measurement of S100B allows the reduction of CCT by approximately $30 \%$ in older patients with MHI who fulfill the described criteria. Of our CCT-negative patients, $64.7 \%$ were S100B positive. The low PPV (9.4\%) means that a positive S100B cannot be used for further clinical decision making.

A few limitations concerning our results should be considered. First, we have no data concerning the outcome of our CCT-negative patients beyond the observation period. The median interval between injury and CCT was 15:40 hours, and the median observation period was 31:37 hours. We cannot rule out hemorrhages that might have occurred after the end of observation. However, neurological deterioration would have been a reason for readmission. Second, the history of antiplatelet drug use is not very reliable in older patients, and aspirin resistance is a frequently observed multifactorial phenomenon. ${ }^{35}$ The effects of aspirin and clopidogrel have been shown to be highly variable between individuals. Furthermore, medication interaction, absorption, and metabolism are factors that can significantly impact bleeding risk and platelet function. ${ }^{19}$ Platelet function testing was not done in the context of our study.

\section{Conclusions}

We can conclude that S100B levels below $0.105 \mu \mathrm{g} / \mathrm{L}$ can accurately predict a normal CCT scan after MHI in older patients and those on antiplatelet medication (lowdose aspirin and/or clopidogrel). Combining conventional clinical decision criteria with measurement of S100B provides useful information for the management of MHI in these patients and leads to the reduction of CCT scans and admissions for observation by approximately $30 \%$. 
In times of increasing concern about overdiagnosis and overtreatment, this is of special importance with regard to radiation-associated health risks and financial burden.

\section{References}

1. af Geijerstam JL, Britton M: Mild head injury - mortality and complication rate: meta-analysis of findings in a systematic literature review. Acta Neurochir (Wien) 145:843-850, 2003

2. Biberthaler P, Linsenmeier U, Pfeifer KJ, Kroetz M, Mussack T, Kanz KG, et al: Serum S-100B concentration provides additional information for the indication of computed tomography in patients after minor head injury: a prospective multicenter study. Shock 25:446-453, 2006

3. Brenner DJ, Hall EJ: Computed tomography-an increasing source of radiation exposure. N Engl J Med 357:2277-2284, 2007

4. Davis PC: Head trauma. AJNR Am J Neuroradiol 28:16191621,2007

5. Efron B, Tibshirani RJ: An Introduction to the Bootstrap. London: Chapman \& Hall, 1993

6. Fabbri A, Servadei F, Marchesini G, Bronzoni C, Montesi D, Arietta L: Antiplatelet therapy and the outcome of subjects with intracranial injury: the Italian SIMEU study. Crit Care 17:R53, 2013

7. Harnan SE, Pickering A, Pandor A, Goodacre SW: Clinical decision rules for adults with minor head injury: a systematic review. J Trauma 71:245-251, 2011

8. Hartholt KA, Van Lieshout EM, Polinder S, Panneman MJ, Van der Cammen TJ, Patka P: Rapid increase in hospitalizations resulting from fall-related traumatic head injury in older adults in The Netherlands 1986-2008. J Neurotrauma 28:739-744, 2011

9. Haydel MJ, Preston CA, Mills TJ, Luber S, Blaudeau E, DeBlieux PM: Indications for computed tomography in patients with minor head injury. N Engl J Med 343:100-105, 2000

10. He H, McDermott MP: A robust method using propensity score stratification for correcting verification bias for binary tests. Biostatistics 13:32-47, 2012

11. Holm L, Cassidy JD, Carroll LJ, Borg J: Summary of the WHO Collaborating Centre for Neurotrauma Task Force on Mild Traumatic Brain Injury. J Rehabil Med 37:137-141, 2005

12. Ingebrigtsen T, Romner B, Marup-Jensen S, Dons M, Lundqvist C, Bellner J, et al: The clinical value of serum S-100 protein measurements in minor head injury: a Scandinavian multicentre study. Brain Inj 14:1047-1055, 2000

13. Jagoda AS, Bazarian JJ, Bruns JJ Jr, Cantrill SV, Gean AD, Howard PK, et al: Clinical policy: neuroimaging and decision making in adult mild traumatic brain injury in the acute setting. Ann Emerg Med 52:714-748, 2008

14. Laribi S, Kansao J, Borderie D, Collet C, Deschamps P, Ababsa R, et al: S100B blood level measurement to exclude cerebral lesions after minor head injury: the multicenter STIC-S100 French study. Clin Chem Lab Med 52:527-536, 2014

15. Mina AA, Knipfer JF, Park DY, Bair HA, Howells GA, Bendick PJ: Intracranial complications of preinjury anticoagulation in trauma patients with head injury. J Trauma 53:668-672, 2002

16. Morton MJ, Korley FK: Head computed tomography use in the emergency department for mild traumatic brain injury: integrating evidence into practice for the resident physician. Ann Emerg Med 60:361-367, 2012

17. Müller K, Townend W, Biasca N, Undén J, Waterloo K, Romner B, et al: S100B serum level predicts computed tomography findings after minor head injury. J Trauma 62:1452-1456, 2007
18. Pandor A, Goodacre S, Harnan S, Holmes M, Pickering A, Fitzgerald P, et al: Diagnostic management strategies for adults and children with minor head injury: a systematic review and an economic evaluation. Health Technol Assess 15:1-202, 2011

19. Patrono C, Baigent C, Hirsh J, Roth G: Antiplatelet drugs: American College of Chest Physicians Evidence-Based Clinical Practice Guidelines (8th edition). Chest 133 (6 Suppl):199S-233S, 2008

20. Peck KA, Sise CB, Shackford SR, Sise MJ, Calvo RY, Sack DI, et al: Delayed intracranial hemorrhage after blunt trauma: are patients on preinjury anticoagulants and prescription antiplatelet agents at risk? J Trauma 71:1600-1604, 2011

21. Rothermundt M, Peters M, Prehn JH, Arolt V: S100B in brain damage and neurodegeneration. Microsc Res Tech 60:614-632, 2003

22. Rothoerl RD, Woertgen C, Holzschuh M, Metz C, Brawanski A: S-100 serum levels after minor and major head injury. $\mathbf{J}$ Trauma 45:765-767, 1998

23. Roudsari B, Psoter KJ, Fine GC, Jarvik JG: Falls, older adults, and the trend in utilization of CT in a level I trauma center. AJR Am J Roentgenol 198:985-991, 2012

24. Ruan S, Noyes K, Bazarian JJ: The economic impact of S$100 \mathrm{~B}$ as a pre-head CT screening test on emergency department management of adult patients with mild traumatic brain injury. J Neurotrauma 26:1655-1664, 2009

25. Smith-Bindman R: Is computed tomography safe? N Engl J Med 363:1-4, 2010

26. Spektor S, Agus S, Merkin V, Constantini S: Low-dose aspirin prophylaxis and risk of intracranial hemorrhage in patients older than 60 years of age with mild or moderate head injury: a prospective study. J Neurosurg 99:661-665, 2003

27. Stiell IG, Wells GA, Vandemheen K, Clement C, Lesiuk H, Laupacis A, et al: The Canadian CT Head Rule for patients with minor head injury. Lancet 357:1391-1396, 2001

28. Tauber M, Koller H, Moroder P, Hitzl W, Resch H: Secondary intracranial hemorrhage after mild head injury in patients with low-dose acetylsalicylate acid prophylaxis. J Trauma 67:521-525, 2009

29. Teasdale G, Jennett B: Assessment of coma and impaired consciousness. A practical scale. Lancet 2:81-84, 1974

30. Thompson HJ, McCormick WC, Kagan SH: Traumatic brain injury in older adults: epidemiology, outcomes, and future implications. J Am Geriatr Soc 54:1590-1595, 2006

31. Townend W, Dibble C, Abid K, Vail A, Sherwood R, Lecky F: Rapid elimination of protein S-100B from serum after minor head trauma. J Neurotrauma 23:149-155, 2006

32. Townend WJ, Guy MJ, Pani MA, Martin B, Yates DW: Head injury outcome prediction in the emergency department: a role for protein S-100B? J Neurol Neurosurg Psychiatry 73:542-546, 2002

33. Undén J, Ingebrigtsen T, Romner B: Scandinavian guidelines for initial management of minimal, mild and moderate head injuries in adults: an evidence and consensus-based update. BMC Med 11:50, 2013

34. Undén J, Romner B: Can low serum levels of S100B predict normal CT findings after minor head injury in adults?: an evidence-based review and meta-analysis. J Head Trauma Rehabil 25:228-240, 2010

35. Vaturi M, Vaduganathan M, Bental T, Solodky A, Kornowski $\mathrm{R}$, Lev EI: Relation of aspirin response to age in patients with stable coronary artery disease. Am J Cardiol 112:212-216, 2013

36. Williams DH, Levin HS, Eisenberg HM: Mild head injury classification. Neurosurgery 27:422-428, 1990

37. Yuan MK, Tsai DC, Chang SC, Yuan MC, Chang SJ, Chen $\mathrm{HW}$, et al: The risk of cataract associated with repeated head and neck CT studies: a nationwide population-based study. AJR Am J Roentgenol 201:626-630, 2013 
38. Zongo D, Ribéreau-Gayon R, Masson F, Laborey M, Contrand B, Salmi LR, et al: S100-B protein as a screening tool for the early assessment of minor head injury. Ann Emerg Med 59:209-218, 2012

\section{Author Contributions}

Conception and design: Thaler, Schmidsfeld, Gleiss, Mousavi. Acquisition of data: Thaler, Schmidsfeld, Pusch, Pienaar, Wunderer, Pittermann. Analysis and interpretation of data: Thaler, Schmidsfeld, Pienaar, Valenta, Gleiss, Mousavi. Drafting the article: Thaler, Schmidsfeld, Pienaar, Gleiss. Critically revising the article: all authors. Reviewed submitted version of manuscript: all authors. Approved the final version of the manuscript on behalf of all authors: Thaler. Statistical analysis: Gleiss. Study supervision: Fialka, Mousavi.

\section{Supplemental Information}

Previous Presentation

Portions of this work were presented in poster form at the Congress on Geriatric Traumatology in Stuttgart, Germany, on March $28,2014$.

\section{Correspondence}

Heinrich W. Thaler, Trauma Hospital Meidling, Kundratstrasse 37, A-1120 Vienna, Austria. email: heinrich.thaler@auva.at. 